\title{
Travelers' health problems and behavior: prospective study with post-travel follow-up
}

\author{
Katri Vilkman ${ }^{1,2}$, Sari H. Pakkanen ${ }^{1}$, Tinja Lääveri' ${ }^{2}$, Heli Siikamäki ${ }^{2}$ and Anu Kantele $2,3,4,5^{*}$ (D)
}

\begin{abstract}
Background: The annual number of international tourist arrivals has recently exceeded one billion, yet surprisingly few studies have characterized travelers' behavior, illness, and risk factors in a prospective setting. Particularly scarce are surveys of data spanning travel, return, and follow-up of the same cohort.

This study examines behavior and illness among travelers while abroad, after return home, and at follow-up. Patterns of behavior connected to type of travel and illness are characterized so as to identify risk factors and provide background data for pre-travel advice.

Methods: Volunteers to this prospective cohort study were recruited at visits to a travel clinic prior to departure. Data on the subjects' health and behavior were collected by questionnaires before and after journeys and over a three-week follow-up. In addition, the subjects were asked to fill in health diaries while traveling.

Results: The final study population consisted of 460 subjects, $79 \%$ of whom reported illness during travel or on arrival: $69 \%$ had travelers' diarrhea (TD), $17 \%$ skin problems, $17 \%$ fever, $12 \%$ vomiting, $8 \%$ respiratory tract infection, $4 \%$ urinary tract infection, $2 \%$ ear infection, $4 \%$ gastrointestinal complaints other than TD or vomiting, and $4 \%$ other symptoms. Of all subjects, $10 \%$ consulted a doctor and $0.7 \%$ were hospitalized; $18 \%$ took antimicrobials, with TD as the most common indication (64\%). Ongoing symptoms were reported by $25 \%$ of all travelers upon return home.

During the three-week follow-up (return rate $51 \%$ ), $32 \%$ of respondents developed new-onset symptoms, $20 \%$ visited a doctor and $1.7 \%$ were hospitalized.

Factors predisposing to health problems were identified by multivariable analysis: certain regions (Southern Asia, South-Eastern Asia, and Eastern Africa), female gender, young age, and long travel duration.

Conclusions: Despite proper preventive measures like vaccinations, malaria prophylaxis, and travel advice, the majority of our subjects fell ill during or after travel. As the symptoms mostly remained mild, health care services were seldom needed. Typical traveler profiles were identified, thereby providing a tool for pre-travel advice. The finding that one third reported new-onset illness during follow-up attests to the importance of advising clients on potential post-travel health problems already during pre-travel visits.
\end{abstract}

Keywords: Travel, Travelers' health, Travelers' behavior, Travelers' diarrhea, Malaria, Antimalarials, Risk factors, Vaccinations, Antimicrobials

\footnotetext{
* Correspondence: anu.kantele@hus.fi

${ }^{2}$ Inflammation Center, Clinic of Infectious Diseases, Helsinki University

Hospital and University of Helsinki, Aurora Hospital, Nordenskiöldinkatu 20,

(P.O. Box 348), Helsinki, Finland

${ }^{3}$ Institute of Clinical Medicine, University of Helsinki, Helsinki, Finland

Full list of author information is available at the end of the article
} 


\section{Background}

According to travel records for 2015, the number of international tourist arrivals exceeded one billion, and half a billion people headed to emerging economies [1]. Less than half of the visitors to poor regions have been shown to seek pre-travel health advice, as exemplified by only $15 \%$ of Canadians visiting hepatitis A endemic countries [2] and $31 \%$ of Australasians traveling to Asia, Africa, or South America [3]. This is surprising, considering the high morbidity rates (64-70 \%) reported for visitors to developing regions $[4,5]$.

Investigations addressing travelers' health have generally been retrospective and/or conducted among those seeking medical care after their journeys [6-21]. Not many prospective studies focus on the spectrum of travelers' diseases [4, 5, 22-27]. Among these we found only one that looks at the same cohort during and after travel and also includes a post-travel follow-up [4]. The principal findings of the prospective studies are mildness of symptoms $[4,22]$ and small proportion of those falling ill who seek medical care: $2-33 \%$ of the total study population visit a physician and $0.1-4 \%$ are hospitalized $[4,5,22,24,25,27]$ during travel. After returning home, $9-20 \%$ of all travelers have been reported to see a doctor $[4,5,22,25]$ and $0.0-1.0 \%$ to be hospitalized [4, 22, 27]. Even though those with the most severe symptoms probably seek medical care, it should be noted that they represent only the tip of the iceberg among travelers falling ill. To get a comprehensive view of travel-associated health problems, prospective study designs should be employed for collecting data on illness while abroad, after return, and at follow-up.

To examine the health problems of travelers overseas, we recently conducted a nation-wide study of a large Finnish database provided by an assistance organization [21]. While these data presumably cover cases with the most severe symptoms, we sought to complete them with a prospective study that would comprise even mild illness. By including a post-travel follow-up, we extended the research to symptoms not developing until after return.

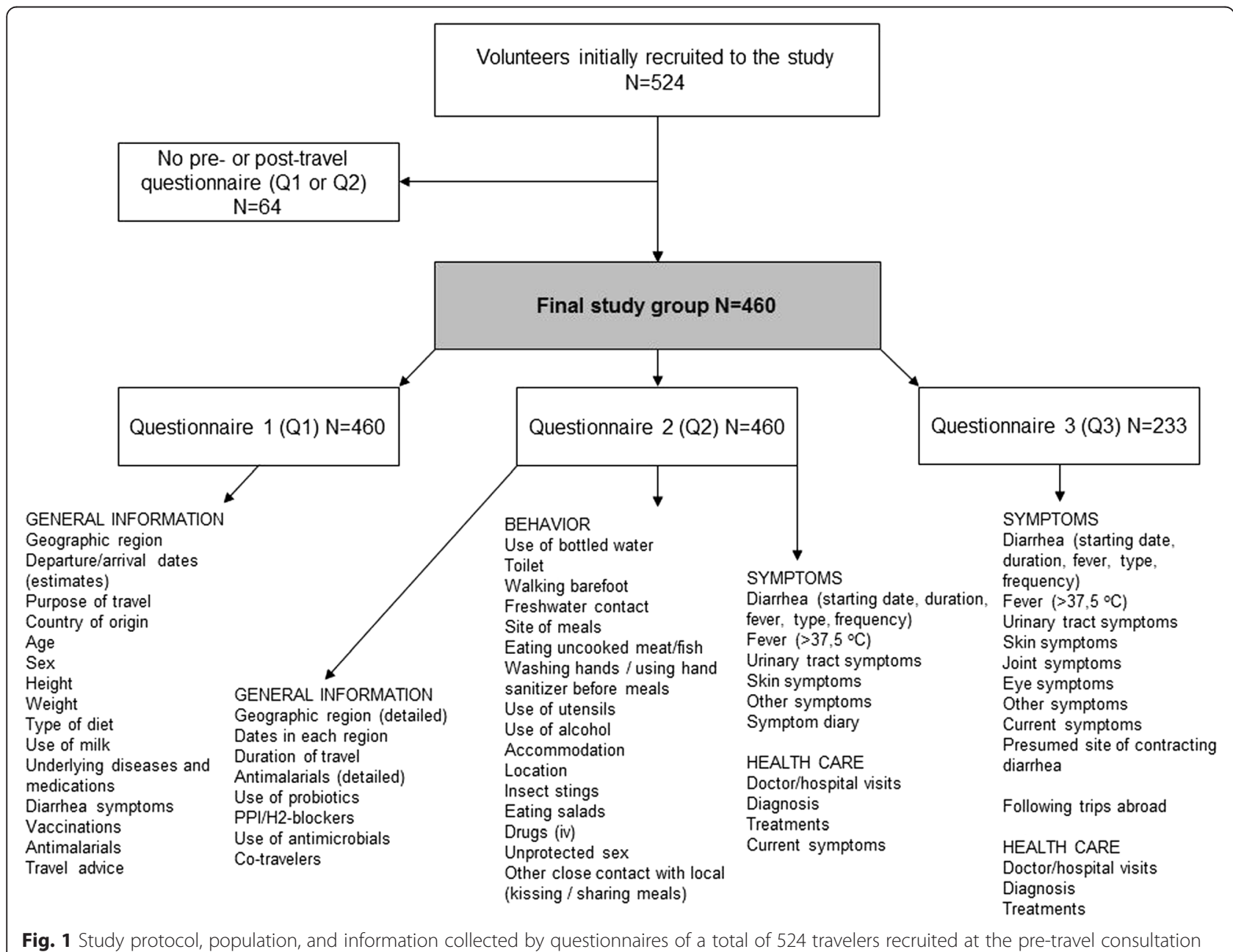


Table 1 Demographics, travel information, and destinations of 460 travelers recruited at the travel clinic during their pre-travel visit, all staying outside the Nordic countries for more than four days and less than 6 months. The data are given as numbers of cases and their proportions

\begin{tabular}{|c|c|c|}
\hline & Number & $\left(\%^{\mathrm{a}}\right)$ \\
\hline \multicolumn{3}{|l|}{ Sex (missing data $=0$ ) } \\
\hline Male & 173 & (38) \\
\hline Female & 287 & (62) \\
\hline Age, median (IQR) (missing data =0) & $35(27.0-54.0)$ & \\
\hline 0-17, children & 35 & (8) \\
\hline 18-35, young adults & 196 & (43) \\
\hline 36-55, middle aged & 120 & (26) \\
\hline $56-$, older travelers & 109 & (24) \\
\hline Underlying diseases (missing data $=0$ ) & 192 & $(42)$ \\
\hline Asthma, atopy and/or allergy & 56 & (12) \\
\hline Hypertension & 51 & (11) \\
\hline Psychiatric disorder or medication & 25 & (5) \\
\hline Cardiovascular disease & 14 & (3) \\
\hline Gl disorder ${ }^{b}$ & 13 & (3) \\
\hline Neurological disorder & 12 & (3) \\
\hline DM & 9 & (2) \\
\hline Rheumatic disease & 6 & (1) \\
\hline Other disease and conditions & 78 & (17) \\
\hline $\begin{array}{l}\text { Contraceptives or postmenopausal hormone } \\
\text { replacement therapy }\end{array}$ & 53 & (12) \\
\hline $\begin{array}{l}\text { Duration of travel, median in days (IQR) } \\
\text { (missing data }=2 \text { ) }\end{array}$ & $16(12.8-27.3)$ & \\
\hline 1 week or less (missing data $=0$ ) & 27 & (6) \\
\hline More than a week and less than a month & 334 & (73) \\
\hline $1-5$ months & 99 & (22) \\
\hline \multicolumn{3}{|l|}{ Purpose of travel (missing data $=1$ ) } \\
\hline Vacation & 383 & (83) \\
\hline Business & 38 & (8) \\
\hline Other/multiple ${ }^{c}$ & 38 & (8) \\
\hline \multicolumn{3}{|l|}{ Geographic region (missing data $=0)^{d}$} \\
\hline South-Eastern Asia & 107 & (23) \\
\hline Eastern Africa & 96 & $(21)$ \\
\hline Western Africa, Middle Africa & 86 & (19) \\
\hline Southern Asia & 68 & (15) \\
\hline Latin America and the Caribbean & 41 & (9) \\
\hline Southern Africa & 26 & (6) \\
\hline Europe, Northern America & 15 & (3) \\
\hline
\end{tabular}

Table 1 Demographics, travel information, and destinations of 460 travelers recruited at the travel clinic during their pre-travel visit, all staying outside the Nordic countries for more than four days and less than 6 months. The data are given as numbers of cases and their proportions (Continued)

Northern Africa, Western Asia
Eastern Asia, Central Asia
a Proportion of positive among those from whom information was available
b Crohn's disease, irritable bowel syndrome, primary biliary cirrhosis,
diverticulosis, microscopic colitis, celiac disease
c 14 (3\%) travelers reported two or more purposes of travel
d Given according to primary destination. Categorized into three subgroups:
Asia (Western Asia, South-Eastern Asia, Southern Asia, Eastern Asia, and Central
Asia), Africa (Eastern Africa, Western Africa, Middle Africa, Southern Africa, and
Northern Africa), and the others (Latin America and the Caribbean, Europe,
North America)

\section{Methods}

Volunteers and study design

Volunteers to this prospective study cohort were enrolled at the Travel Clinic of Aava Medical Centre among travelers planning a journey outside the Nordic countries for a minimum of four days and a maximum of six months (Fig. 1). The only exclusion criterion was non-compliance in returning questionnaires. The subjects were recruited among consecutive clients at pretravel appointments between December 2008 and February 2010. At the initial visit, they filled out a pretravel questionnaire (Q1), on return home a post-travel questionnaire (Q2), and about three weeks later a follow-up questionnaire (Q3). Those who failed to return both Q1 and Q2 were excluded. In addition to the questionnaires, the subjects were asked to complete a structured diary on a voluntary basis. All volunteers were given pre-travel advice by a health care professional.

We have earlier reported risk factors for acquiring resistant intestinal microbes in the same population [28], and, data on diarrheal pathogens, first in our methodological investigation [29] and, recently, in an etiological study [30].

\section{Questionnaires}

The questionnaires consisted of 134 multiple-choice or open-ended questions: Q1 comprised 47, Q2 60, and Q3 27 questions. These questionnaires were modified from a set of survey questions routinely used for more than 10 years for ill travelers admitted to the Clinic of Infectious Diseases at $\mathrm{HUCH}$. All items covered are listed in Fig. 1. The structured diary contained more specific questions on duration and severity of symptoms, and use of antibiotics.

\section{Definitions}

Subjects were classified as ill if they reported symptoms implying health problems which could be travel-related. 
Diarrhea was defined according to WHO criteria, i.e. passage of 3 or more loose or liquid stools per day, or more frequently than is normal for the individual (World Health Organization [31]). At follow-up, symptoms which had set in more than two days after a journey and could be travel-related were categorized as newly onset.

\section{Destinations}

The countries visited were grouped into nine geographic regions (UN categorization, modified [32]): Southern Asia, South-Eastern Asia, Eastern Asia and Central Asia, Southern Africa, Eastern Africa, Western Africa and Middle Africa, Northern Africa and Western Asia, Latin America and the Caribbean, and Europe and Northern America (Table 1). The destination with the greatest health risk was considered primary for subjects traveling to several places. Here we used a rating based on the risk map drawn up by International SOS (Additional file 1: Figure S1 HealthMap 2010) which takes into account a range of factors: the standard of local medical and dental care, access to prescription drugs, the possible prevalence of serious infectious diseases, and known cultural, linguistic and administrative barriers.

\section{Statistics}

Univariable, bivariable and multivariable models were used. The p-value of Pearson Chi-square tests and Fisher's exact test $<0.05$ was considered statistically significant. If bivariable p-value was less than 0.10 , the factor was chosen to the multivariable logistic regression model, and its adjusted odds ratios and $95 \%$ confidence intervals were calculated. Missing data were assumed to be missing at random (MAR) and missing values were imputed using multiple imputations in SPSS. The statistical analyzes were carried out with SPSS Statistics (version 22.0.0.2, IBM Corp., USA).

\section{Results}

\section{Cohort population and prophylactic measures}

Of the 524 initially recruited travelers, $88 \%$ completed both pre- and post-travel questionnaires. The final study population thus consisted of 460 volunteers (Fig. 1, Table 1). A total of 233 (51\%) returned follow-up questionnaires and 295 (64\%) filled in diaries. An underlying disease or condition was recorded for 192 (42\%)(Table 1), more frequently among the oldest subjects (56- years, $73 \% ; p<0.001)$, and females (45\%; $p=0.046)$ than in other age groups $(0-55$ years, $23-38 \%)$ or among males (36\%). Data on vaccinations are presented in Table 2 and on antimalarials in Table 3.

\section{Travel information and behavior}

The 460 travelers visited 77 countries altogether (UN definition); India (64), Thailand (60), Gambia (50), Tanzania (48), and Kenya (40) ranking as their most popular destinations. Overall 662 country visits were made (average 1.45 per person; range 1-8). The 30 most favored countries accounted for 560 journeys ( $85 \%)$; only three destinations were in developed regions (UN definition [33]): USA (8), Spain (7), and the Netherlands (5).

Table 2 Numbers and proportions of travelers $(n=460)$ vaccinated before the travel clinic appointment or at consultation. The data are given as numbers and percentages of those vaccinated or considered protected according to Finnish recommendations

\begin{tabular}{llll}
\hline & $\begin{array}{l}\text { Protected before } \\
\text { consultation, } N\left(\%^{\mathrm{a}}\right)\end{array}$ & $\begin{array}{l}\text { Vaccinated at } \\
\text { consultation, } N\left(\%{ }^{\mathrm{a}}\right)\end{array}$ & $\begin{array}{l}\text { Protected according to recommendations given for the } \\
\text { region visited, } N\left(\%^{\mathrm{a}}\right)\end{array}$ \\
\hline Tetanus (missing data $=4)$ & $353(77)$ & $96(21)$ & $449(98)$ \\
MMR (missing data $=43)$ & $393(94)$ & $21(5)$ & $414(99)$ \\
Hepatitis A (missing data $=3)$ & $325(71)$ & $130(28)$ & $440(100)$ \\
Hepatitis B (missing data $=4)$ & $178(39)$ & $124(27)$ & $72(73)$ \\
Yellow Fever (missing data $=2)$ & $112(24)$ & $153(33)$ & $182(96)$ \\
Polio (missing data $=4)^{\text {b }}$ & $140(31)$ & $84(18)$ & $104(63)$ \\
$\begin{array}{l}\text { Typhoid fever (missing data }= \\
\text { 3) }\end{array}$ & $38(8)$ & $63(14)$ & $47(65)$ \\
oral & $16(4)$ & $59(13)$ & $18(53)$ \\
injected & $22(5)$ & $4(1)$ & \\
Japanese encephalitis (missing \\
data = 3)
\end{tabular}

a Proportion of positive among those from whom information was available

${ }^{b}$ Part of the volunteers may have chosen to skip polio vaccination during appointment at the travel clinic and, instead, get it from their health center where they are vaccinated free of charge since polio is included in the Finnish national immunization program

'Despite advice many declined prophylaxis because of high price

${ }^{d}$ Includes cholera, meningococcal, and rabies vaccinations 
Table 3 Medications taken by 460 Finnish travelers during the trip. The medications are categorized according to indication. The data are presented as numbers and percentages of travelers

\begin{tabular}{|c|c|c|}
\hline & Number & $\left(\%^{a}\right)$ \\
\hline $\begin{array}{l}\text { Prophylactic antimalarial use among those } \\
\text { recommended (missing data }=6 \text { ) }\end{array}$ & 289 & (98) \\
\hline Atovaquone and proguanil & 135 & (30) \\
\hline Mefloquine & 70 & $(15)$ \\
\hline Doxycycline & 55 & (12) \\
\hline Chloroquine & 25 & (6) \\
\hline Other / not known & 2 & (0) \\
\hline Changed drug ${ }^{b}$ & 2 & (0) \\
\hline Failure in use ${ }^{c}$ & 7 & (2) \\
\hline Antimicrobials (other than doxicycline) (missing data $=0)^{d}$ & 81 & $(18)$ \\
\hline Fluoroquinolone & 53 & $(12)$ \\
\hline Macrolide & 9 & (2) \\
\hline Nitroimidazole (metronidazole or tinidatzole) & 7 & (2) \\
\hline Amoxicillin & 5 & (1) \\
\hline Tetracycline & 1 & (0) \\
\hline Other / not known & 25 & (5) \\
\hline Two or more antimicrobials (without doxycycline) & 15 & (3) \\
\hline Treatment for malaria (missing data $=0$ ) ${ }^{e}$ & 4 & (1) \\
\hline Gastrointestinal medication (missing data $=0$ ) & 127 & $(28)$ \\
\hline Loperamide & 115 & $(25)$ \\
\hline Medicinal charcoal & 7 & (2) \\
\hline Other (laxative, spasmolyte, co-phenotrope) & 10 & (2) \\
\hline Two or more gastrointestinal medications & 5 & (1) \\
\hline Analgesic (missing data $=0$ ) & 123 & $(27)$ \\
\hline NSAID & 67 & (15) \\
\hline Paracetamol & 59 & (13) \\
\hline Other / not known & 16 & (3) \\
\hline Two or more analgesics & 18 & (4) \\
\hline Antiemetic (missing data $=0$ ) & 4 & (1) \\
\hline PPI or antacid (missing data $=4$ ) & 36 & (8) \\
\hline PPI & 26 & (6) \\
\hline Antacid (salts/H2) & 10 & (2) \\
\hline Probiotics and prebiotics (missing data $=3)^{\mathrm{f}}$ & 270 & (59) \\
\hline Starting before travel & 11 & (2) \\
\hline Using only during travel & 123 & $(27)$ \\
\hline Starting before and using during travel & 136 & (30) \\
\hline
\end{tabular}

a Total use of all travelers among those from whom information was available

${ }^{\mathrm{b}}$ Each of these changed the medication from doxycycline to mefloquine

' Did not use as recommended ( 2 did not take at all, 1 stopped because of adverse effects, 4 used irregularly): atovaquone/proguanil (4), doxycycline (2), mefloquine (1); 10 had prescription but did not enter malaria area; 33 traveled to endemic countries yet visited only low prevalence areas

d 52 (11\% of all) used antimicrobials for TD

${ }^{\text {e }}$ All in Africa, three of four were tested; two negative, one positive

${ }^{f}$ If only product mentioned, presumed to be used while traveling
Uncooked meat/fish (Table 4) was eaten more frequently by young adults (18-35 years, $17 \%)$ and the middle-aged (36-55 years, $13 \%)$ than children $(0-17$ years, $6 \%)$ and older travelers (56- years, $6 \%)(p=$ 0.016). Young adults proved more likely than the others to have freshwater contact, neglect hand washing, and not to shun salads or eating without utensils. Men consumed more alcohol than women $(p<0.001)$.

\section{Traveler profiles}

Traveler profiles by age

The median duration of travel was 23 days for the young adults and 15 days for others (age group vs travel duration group $p<0.001$ ). Young adults and children stayed most often in guest houses, while the others preferred hotel accommodation (Table 4).

\section{Traveler profiles by destinations}

To obtain data on typical travelers to the two most preferred geographic regions, Africa and Asia, the destinations were categorized into three subgroups: Africa $(n=$ $212)$, Asia $(n=192)$, and others $(n=56)$. Their characteristics are presented in Table 4. In bivariable analysis, distribution of age and duration of journey differed between those heading for Africa and those favoring Asia. The median age was higher for those visiting Africa, and the journey was shorter. Likewise, compared to Asia, visitors to Africa had less frequent freshwater contact and ate uncooked meat/fish less often.

\section{Illness}

Factors with $p<0.1$ in the bivariable analyses (Table 5) were included in the multivariable analyses (Table 6). Destination associated strongly with health problems. Females were more predisposed to falling ill. Overall, the risk was greatest at 31.5 years of age. The longer the travel, the greater the risk of contracting an illness - it increased each day by $2.5 \%$. Eating raw meat/fish was associated with healthier travelers.

Geographic regions with demographics and proportions of sick travelers are presented in Fig. 2. Illness rates by geographic regions were found to accord with respective risk ratings by International SOS (Additional file 2: Figure S2).

Health care was sought by $10 \%$ of all subjects and by $13 \%$ of those fallen ill during travel; $10 \%$ and $13 \%$ visited a physician and $0.7 \%$ and $0.9 \%$, respectively, were hospitalized (Table 7). The most common reason for consulting health care professionals while abroad was TD $-4 \%$ of all travelers visited a doctor, and $0.4 \%$ were subsequently admitted to hospital because of this illness.

During follow-up, about one-third reported new-onset health problems (Table 7). Their most common single 
Table 4 Traveler profiles presented by demographics (destination, sex, age, duration of travel, purpose of travel) with travel behavior in bivariable analysis for 460 travelers. Results are given in percentages with the largest group shown in bold

\begin{tabular}{|c|c|c|c|c|c|c|c|c|c|c|c|c|c|c|c|c|c|c|c|c|c|}
\hline & \multirow{2}{*}{$\begin{array}{l}\text { Number } \\
(\%)\end{array}$} & \multicolumn{4}{|c|}{ Destination \% } & \multicolumn{3}{|c|}{ Sex \% } & \multicolumn{5}{|c|}{ Age group (years) \% } & \multicolumn{4}{|c|}{ Duration of travel (days) \% } & \multicolumn{4}{|c|}{ Purpose of travel \% } \\
\hline & & Africa & Asia & other & $p$-value & male & female & $p$-value & $\begin{array}{l}0- \\
17\end{array}$ & $\begin{array}{l}18- \\
35\end{array}$ & $\begin{array}{l}36- \\
55\end{array}$ & $56-$ & $p$-value & $1-7$ & $\begin{array}{l}8- \\
29\end{array}$ & $\begin{array}{l}30- \\
160\end{array}$ & $p$-value & vacation & business & other & $p$-value \\
\hline $\begin{array}{l}\text { Age group } \\
(\text { missing data }=0)\end{array}$ & & & & & $<0.001^{*}$ & & & 0.833 & & & & & & & & & $<0.001^{*}$ & & & & 0.056 \\
\hline $0-17$ & $35(8)$ & 8 & 7 & 11 & & 8 & 7 & & & & & & & 30 & 7 & 3 & & 9 & 0 & 3 & \\
\hline $18-35$ & $196(43)$ & 32 & 55 & 41 & & 43 & 42 & & & & & & & 22 & 36 & 72 & & 42 & 37 & 61 & \\
\hline $36-55$ & $120(26)$ & 30 & 23 & 23 & & 27 & 25 & & & & & & & 33 & 29 & 13 & & 26 & 39 & 16 & \\
\hline $56-$ & $109(24)$ & 31 & 15 & 25 & & 21 & 25 & & & & & & & 15 & 28 & 12 & & 24 & 24 & 21 & \\
\hline $\begin{array}{l}\text { Duration of travel, days } \\
\text { (missing data }=0 \text { ) }\end{array}$ & & & & & $<0.001^{*}$ & & & 0.473 & & & & & $<0.001^{*}$ & & & & & & & & $<0.001^{d *}$ \\
\hline $1-7$ & $27(6)$ & 2 & 6 & 20 & & 7 & 5 & & 23 & 3 & 8 & 4 & & & & & & 6 & 13 & 0 & \\
\hline $8-29$ & $334(73)$ & 81 & 63 & 73 & & 72 & 73 & & 69 & 61 & 82 & 85 & & & & & & 79 & 53 & 29 & \\
\hline $30-160$ & $99(22)$ & 17 & 31 & 7 & & 20 & 22 & & 9 & 36 & 11 & 11 & & & & & & 15 & 34 & 71 & \\
\hline $\begin{array}{l}\text { Purpose of travel } \\
\left(_{\text {missing data }=1)^{\text {a }}}\right.\end{array}$ & & & & & $0.034^{d *}$ & & & $0.002^{*}$ & & & & & 0.056 & & & & $<0.001^{d *}$ & & & & \\
\hline vacation & $383(84)$ & 78 & 88 & 91 & & 86 & 82 & & 97 & 81 & 83 & 84 & & 81 & 91 & 60 & & & & & \\
\hline business & $37(8)$ & 12 & 5 & 4 & & 11 & 7 & & 0 & 7 & 13 & 8 & & 19 & 6 & 13 & & & & & \\
\hline other/multiple & $35(8)$ & 10 & 7 & 5 & & 3 & 11 & & 3 & 12 & 5 & 7 & & 0 & 3 & 27 & & & & & \\
\hline $\begin{array}{l}\text { Accommodation } \\
(\text { missing data }=11)^{\mathrm{b}}\end{array}$ & & & & & $<0.001^{*}$ & & & $0.008^{*}$ & & & & & $<0.001^{*}$ & & & & $<0.001^{*}$ & & & & $<0.001^{*}$ \\
\hline hotel & $188(42)$ & 39 & 40 & 61 & & 46 & 40 & & 34 & 31 & 50 & 55 & & 85 & 46 & 15 & & 44 & 45 & 18 & \\
\hline home of a local & $71(16)$ & 23 & 9 & 14 & & 9 & 20 & & 26 & 16 & 11 & 17 & & 15 & 12 & 29 & & 12 & 16 & 50 & \\
\hline guest house & $190(42)$ & 39 & 52 & 25 & & 45 & 41 & & 40 & 53 & 39 & 28 & & 0 & 42 & 56 & & 44 & 39 & 32 & \\
\hline $\begin{array}{l}\text { Location } \\
(\text { missing data }=22)^{b}\end{array}$ & & & & & $<0.001^{*}$ & & & 0.083 & & & & & 0.088 & & & & $<0.001^{*}$ & & & & 0.494 \\
\hline city & $143(33)$ & 24 & 34 & 57 & & 28 & 36 & & 44 & 27 & 36 & 38 & & 88 & 31 & 23 & & 33 & 38 & 25 & \\
\hline countryside/jungle & $295(67)$ & 76 & 66 & 43 & & 72 & 64 & & 56 & 73 & 66 & 62 & & 12 & 69 & 77 & & 67 & 62 & 75 & \\
\hline 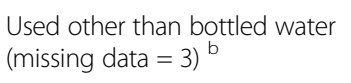 & $25(5)$ & 8 & 3 & 5 & 0.144 & 6 & 5 & 0.499 & 3 & 4 & 8 & 6 & 0.373 & 0 & 5 & 7 & 0.336 & 5 & 11 & 5 & $0.379^{d}$ \\
\hline $\begin{array}{l}\text { Ate uncooked meat/fish } \\
\text { (missing data }=4 \text { ) }\end{array}$ & $58(13)$ & 9 & 16 & 18 & $0.045^{*}$ & 11 & 14 & 0.446 & 6 & 17 & 13 & 6 & $0.016^{*}$ & 4 & 12 & 18 & 0.080 & 13 & 13 & 11 & $0.961^{d}$ \\
\hline Ate salads (missing data $=26$ ) & $341(79)$ & 77 & 78 & 85 & 0.427 & 79 & 78 & 0.904 & 59 & 83 & 81 & 74 & $0.009^{*}$ & 69 & 78 & 83 & 0.288 & 80 & 70 & 78 & 0.404 \\
\hline Diet (missing data = 159) & & & & & 0.087 & & & $0.018^{*}$ & & & & & $0.010^{*}$ & & & & 0.288 & & & & $1.000^{\mathrm{d}}$ \\
\hline omnivore & $274(91)$ & 92 & 88 & 100 & & 96 & 88 & & 100 & 85 & 92 & 97 & & 100 & 92 & 88 & & 91 & 91 & 93 & \\
\hline vegetarian & $27(9)$ & 8 & 12 & 0 & & 4 & 12 & & 0 & 15 & 8 & 3 & & 0 & 8 & 13 & & 9 & 9 & 7 & \\
\hline
\end{tabular}


Table 4 Traveler profiles presented by demographics (destination, sex, age, duration of travel, purpose of travel) with travel behavior in bivariable analysis for 460 travelers. Results are given in percentages with the largest group shown in bold (Continued)

\begin{tabular}{|c|c|c|c|c|c|c|c|c|c|c|c|c|c|c|c|c|c|c|c|c|c|}
\hline $\begin{array}{l}\text { Used milk as part of diet } \\
\text { (missing data }=161 \text { ) }\end{array}$ & $293(98)$ & 99 & 97 & 96 & $0.226^{d}$ & 99 & 97 & $0.426^{d}$ & 96 & 98 & 99 & 97 & $0.599^{d}$ & 100 & 99 & 96 & $0.347^{d}$ & 98 & 100 & 100 & $1.000^{d}$ \\
\hline $\begin{array}{l}\text { Site of meals } \\
(\text { missing data }=12)^{b}\end{array}$ & & & & & $<0.001^{*}$ & & & 0.099 & & & & & $<0.001^{*}$ & & & & 0.660 & & & & $0.042^{*}$ \\
\hline restaurant more than $50 \%$ & $372(83)$ & 70 & 96 & 87 & & 87 & 81 & & 53 & 86 & 86 & 83 & & 89 & 83 & 81 & & 84 & 83 & 68 & \\
\hline $\begin{array}{l}\text { own household and } \\
\text { sometimes elsewhere }\end{array}$ & $76(17)$ & 30 & 4 & 13 & & 13 & 19 & & 47 & 14 & 14 & 17 & & 11 & 17 & 19 & & 16 & 17 & 32 & \\
\hline $\begin{array}{l}\text { Alcohol consumption } \\
\left(_{\text {missing data }=67)^{c}}\right.\end{array}$ & & & & & 0.112 & & & $<0.001^{*}$ & & & & & $0.001^{*}$ & & & & 0.117 & & & & $0.025^{*}$ \\
\hline $0-2$ units per day & $281(61)$ & 72 & 74 & 59 & & 61 & 78 & & 100 & 70 & 64 & 71 & & 65 & 69 & 80 & & 70 & 64 & 91 & \\
\hline 3- units per day & $112(24)$ & 28 & 26 & 41 & & 39 & 22 & & 0 & 30 & 36 & 29 & & 35 & 31 & 20 & & 30 & 36 & 9 & \\
\hline $\begin{array}{l}\text { Did not always use utensils } \\
\text { (missing data }=23 \text { ) }\end{array}$ & $126(29)$ & 28 & 34 & 18 & 0.070 & 28 & 29 & 0.877 & 39 & 40 & 24 & 10 & $<0.001^{*}$ & 17 & 22 & 55 & $<0.001^{*}$ & 26 & 39 & 49 & $0.004^{*}$ \\
\hline $\begin{array}{l}\text { Did not wash hands } \\
\text { always/often (missing data }=13 \text { ) }\end{array}$ & $61(14)$ & 10 & 16 & 18 & 0.147 & 17 & 12 & 0.138 & 9 & 21 & 9 & 7 & $0.001^{*}$ & 11 & 13 & 17 & 0.573 & 14 & 14 & 11 & 0.840 \\
\hline $\begin{array}{l}\text { No WC as a toilet } \\
\text { (missing data }=7 \text { ) }\end{array}$ & $74(16)$ & 22 & 13 & 5 & $0.004^{*}$ & 17 & 16 & 0.747 & 16 & 19 & 18 & 10 & 0.235 & 0 & 16 & 21 & $0.037^{*}$ & 16 & 14 & 24 & 0.411 \\
\hline $\begin{array}{l}\text { Had fresh water contact } \\
\text { (missing data }=160)\end{array}$ & 109 (36) & 27 & 47 & 28 & $0.001^{*}$ & 38 & 35 & 0.584 & 26 & 50 & 33 & 14 & $<0.001^{*}$ & 6 & 31 & 59 & $<0.001^{*}$ & 37 & 24 & 46 & 0.243 \\
\hline $\begin{array}{l}\text { Walking barefoot often/ } \\
\text { sometimes (missing data }=7 \text { ) }\end{array}$ & $322(71)$ & 64 & 80 & 69 & $0.002^{*}$ & 74 & 69 & 0.326 & 88 & 79 & 68 & 55 & $<0.001^{*}$ & 65 & 70 & 77 & 0.359 & 74 & 59 & 55 & $0.015^{*}$ \\
\hline $\begin{array}{l}\text { Unprotected sex with local } \\
\text { (missing data }=27 \text { ) }\end{array}$ & $8(2)$ & 4 & 1 & 0 & $0.067^{d}$ & 2 & 2 & $1.000^{d}$ & 0 & 1 & 4 & 1 & $0.239^{d}$ & 0 & 2 & 3 & $0.632^{d}$ & 2 & 0 & 3 & $0.587^{d}$ \\
\hline $\begin{array}{l}\text { Other close contact with local } \\
\text { (kissing / sharing meals) } \\
\text { (missing data }=26 \text { ) }\end{array}$ & $82(19)$ & 23 & 17 & 9 & 0.055 & 17 & 20 & 0.336 & 18 & 25 & 19 & 8 & $0.007^{*}$ & 8 & 15 & 36 & $<0.001^{*}$ & 17 & 24 & 33 & $0.039^{*}$ \\
\hline $\begin{array}{l}\text { Had insect stings } \\
\text { (missing data }=22 \text { ) }\end{array}$ & 335 (76) & 75 & 81 & 67 & 0.058 & 75 & 77 & 0.657 & 59 & 88 & 75 & 62 & $<0.001^{*}$ & 19 & 77 & 93 & $<0.001^{*}$ & 76 & 65 & 91 & $0.032^{*}$ \\
\hline
\end{tabular}

a $14(3 \%)$ reported two or more purposes of travel

${ }^{b}$ Categorized according to lowest standard

${ }^{c}$ Missing data for children under 16 years was replaced with 0 alcohol servings per day

${ }^{\mathrm{d}}$ Fisher's exact test used

* $P$-value less than 0.05 
Table 5 Risk factors of contracting illness while traveling / on arrival among 460 travelers. Values are given for proportions of ill travelers with a given risk factor, odds ratios with $95 \%$ confidence intervals, and $p$-values in a bivariable analysis

\begin{tabular}{|c|c|c|c|c|}
\hline & $\begin{array}{l}\text { Contracted } \\
\text { illness }{ }^{\mathrm{a}} \%\end{array}$ & \multicolumn{2}{|c|}{$\begin{array}{l}\text { Odds ratio ( } 95 \% \\
\text { confidence interval) }\end{array}$} & $p$-value \\
\hline Total (missing data $=1$ ) & 79 & & & \\
\hline \multicolumn{5}{|l|}{ Sex (missing data $=0$ ) } \\
\hline Male & 75 & 1.0 & & \\
\hline Female & 81 & 1.5 & $(1.0-2.4)$ & $0.079^{*}$ \\
\hline \multicolumn{4}{|l|}{ Age group (missing data $=0$ ) } & $<0.001^{*}$ \\
\hline $0-17$ & 66 & 1.0 & & \\
\hline $18-35$ & 89 & 4.3 & $(1.9-9.9)$ & 0.001 \\
\hline $36-55$ & 73 & 1.4 & $(0.6-3.2)$ & 0.380 \\
\hline $56-$ & 71 & 1.3 & $(0.6-2.8)$ & 0.582 \\
\hline \multicolumn{4}{|l|}{ Geographic region (missing data $=0$ ) } & $<0.001^{*}$ \\
\hline Europe, Northern America & 20 & 1.0 & & \\
\hline Latin America and the Caribbean & 66 & 7.7 & $(1.9-32.0)$ & 0.005 \\
\hline Northern Africa, Western Asia & 67 & 8.0 & $(1.4-45.8)$ & 0.019 \\
\hline Southern Africa & 62 & 6.4 & $(1.4-28.4)$ & 0.015 \\
\hline Western Africa, Middle Africa & 79 & 14.9 & $(3.8-58.5)$ & $<0.001$ \\
\hline Eastern Africa & 84 & 21.6 & $(5.4-85.8)$ & $<0.001$ \\
\hline Eastern Asia, Central Asia & 56 & 5.0 & $(0.8-31.0)$ & 0.084 \\
\hline South-Eastern Asia & 87 & 26.6 & $(6.7-106.1)$ & $<0.001$ \\
\hline Southern Asia & 91 & 41.3 & $(9.1-188.5)$ & $<0.001$ \\
\hline \multicolumn{4}{|l|}{ Duration of travel, days (missing data $=0$ ) } & $<0.001^{*}$ \\
\hline $1-7$ & 52 & 1.0 & & \\
\hline $8-29$ & 77 & 3.0 & $(1.4-6.7)$ & 0.006 \\
\hline $30-160$ & 94 & 14.4 & $(4.7-44.1)$ & $<0.001$ \\
\hline \multicolumn{4}{|l|}{ Purpose of travel (missing data $=1$ ) } & 0.813 \\
\hline Vacation & 78 & 1.0 & & \\
\hline Business & 82 & 1.2 & $(0.5-2.9)$ & 0.636 \\
\hline Other/multiple ${ }^{\text {b }}$ & 82 & 1.2 & $(0.5-2.9)$ & 0.636 \\
\hline \multicolumn{4}{|l|}{ Accommodation (missing data $=11)^{c}$} & $<0.001^{*}$ \\
\hline Hotel & 68 & 1.0 & & \\
\hline Home of a local & 87 & 3.2 & $(1.5-6.8)$ & 0.003 \\
\hline Guest house & 87 & 3.1 & $(1.8-5.2)$ & $<0.001$ \\
\hline \multicolumn{5}{|l|}{ Location (missing data $=22)^{c}$} \\
\hline City & 71 & 1.0 & & \\
\hline Countryside/jungle & 83 & 2.0 & $(1.3-3.3)$ & $0.003^{*}$ \\
\hline Used other than bottled water (missing data $=3)^{c}$ & 76 & 0.8 & $(0.3-2.2)$ & 0.710 \\
\hline Ate uncooked meat/fish (missing data $=4$ ) & 66 & 0.4 & $(0.2-0.8)$ & $0.006^{*}$ \\
\hline Ate salads (missing data $=26$ ) & 78 & 0.7 & $(0.4-1.2)$ & 0.192 \\
\hline \multicolumn{5}{|l|}{ Diet $($ missing data $=159$ ) } \\
\hline Omnivore & 82 & 1.0 & & \\
\hline Vegetarian & 89 & 1.8 & $(0.5-6.2)$ & 0.353 \\
\hline Used milk as part of diet (missing data $=161$ ) & 83 & 1.0 & $(0.1-8.5)$ & $1.000^{e}$ \\
\hline
\end{tabular}


Table 5 Risk factors of contracting illness while traveling / on arrival among 460 travelers. Values are given for proportions of ill travelers with a given risk factor, odds ratios with $95 \%$ confidence intervals, and $p$-values in a bivariable analysis (Continued)

\begin{tabular}{|c|c|c|c|c|}
\hline \multicolumn{5}{|l|}{ Site of meals (missing data $=12)^{c}$} \\
\hline Restaurant more than $50 \%$ of meals & 79 & 1.0 & & \\
\hline Own household and sometimes elsewhere & 79 & 1.0 & $(0.5-1.8)$ & 0.996 \\
\hline \multicolumn{5}{|l|}{ Alcohol consumption (missing data $=67)^{d}$} \\
\hline $0-2$ units per day & 81 & 1.0 & & \\
\hline 3- units per day & 74 & 0.7 & $(0.4-1.1)$ & 0.102 \\
\hline Did not always use utensils (missing data $=23$ ) & 85 & 1.7 & $(1.0-3.0)$ & $0.058^{*}$ \\
\hline Did not wash hands always/often (missing data = 13) & 72 & 0.6 & $(0.3-1.2)$ & 0.138 \\
\hline No WC as a toilet (missing data $=7$ ) & 85 & 1.6 & $(0.8-3.2)$ & 0.159 \\
\hline Had fresh water contact (missing data $=160$ ) & 84 & 1.7 & $(0.9-3.1)$ & 0.109 \\
\hline Walking barefoot often/sometimes (missing data $=7$ ) & 79 & 1.0 & $(0.6-1.7)$ & 0.990 \\
\hline Unprotected sex with local (missing data $=27$ ) & 100 & 1.0 & $(1.0-1.0)$ & $0.213^{e}$ \\
\hline Other close contact with local (missing data = 26) & 88 & 2.2 & $(1.1-4.4)$ & $0.030^{*}$ \\
\hline Had insect stings (missing data = 22) & 84 & 3.1 & $(1.9-5.1)$ & $<0.001^{*}$ \\
\hline
\end{tabular}

symptom was fever, followed by respiratory tract infection, skin problems, and TD.

\section{Discussion \\ Introduction}

Investigations addressing travelers' health have generally been retrospective and/or conducted on those seeking medical care after their journeys, whereas prospective studies are scarce. Furthermore, research tends to focus on the effects that one or just a few specific factors have on travelers' behavior and illness: destination [4, 26, 27], length of journey [4, 5, 26, 27], purpose of travel [27], gender [4, 5, 26, 27], age [4, 5, 26, 27], risk behavior [5], and particular diseases, such as TD [34-41]. We are not aware of any earlier investigations into all these factors with a single prospective cohort of travelers; only one [4] provides data on the same subjects during and after travel, and at follow-up.

\section{Morbidity during travel and on return}

A central finding of our work is high morbidity rate. Despite efficient prophylactic measures taken before travel - exemplified by our vaccination data - the amount of health problems proved striking: as many as $76 \%$ of our subjects reported illness while abroad, and $25 \%$ still had ongoing symptoms or new complaints within two days after returning home. The overall proportion of our subjects with any symptoms while overseas (76 \%) correspond to that reported for American (64\%; [4]), U.K. (64 \%; [25]), and Israeli travelers (70\%;
[5]), but proved higher than percentages presented for German (10-43 \%; [26, 27]), Swiss (38 \%; [24]), and Swedish travelers (49\%; [23]). As all these investigations are based on questionnaires, the format of the various questions concerning symptoms may account for the differences.

As regards morbidity in different geographic regions, the percentage of illness proved, as expected, significantly smaller for travelers to advanced countries (20\%) than those visiting developing regions (81\%); the rates were highest for visitors to Southern Asia, SouthEastern Asia, and Eastern Africa. TD accounted for the majority of health problems while abroad / after return (69\%). This result accords with several previous studies $[4-6,8,10,21-24,26,27,42-44]$.

\section{Morbidity at follow-up}

While the symptoms of illness mostly set in abroad, the figures proved unexpectedly high also during the followup: new health problems were reported by $32 \%$ of our subjects. This percentage slightly exceeds the result of the only previous study exploring illness on return and at follow-up; the morbidity rate reported by Hill is $26 \%$ [4]. The duration of follow-up may account for the small difference. In our study it was somewhat shorter (three weeks vs two months). But since the subjects were not asked to give exact dates for their symptoms, the onset could afterwards not be limited to 14 days in cases of TD, respiratory illness, and skin disorders, like in Hill's report. 
Table 6 Risk factors of contracting illness while traveling / on return in the final multivariable model after backward selection of

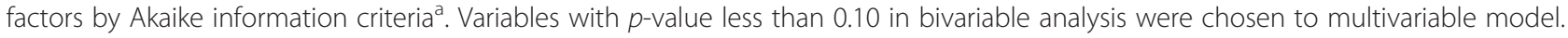
Values are given for proportions with a given risk factor, adjusted odds ratios with $95 \%$ confidence intervals, and $p$-values in multivariable analysis

\begin{tabular}{|c|c|c|c|}
\hline & \multirow{3}{*}{$\begin{array}{l}\text { Proportion of those contracting } \\
\text { illness among travelers with } \\
\text { the given risk factor (\%) }\end{array}$} & \multicolumn{2}{|c|}{ AOR (95 \% Cl) for contracting illness among } \\
\hline & & \multicolumn{2}{|c|}{ travelers with the given risk factor in multivariable } \\
\hline & & analysis with multiple imputations $^{c}$ & $p$-value \\
\hline \multicolumn{4}{|l|}{ Gender } \\
\hline male & 75 & 1.00 & \\
\hline female & 81 & $1.73(1.03-2.91)$ & $0.040^{*}$ \\
\hline Age, years ${ }^{b}$ & N/A & $1.06(0.994-1.14)$ & 0.076 \\
\hline Age, quadratic term ${ }^{b}$ & N/A & $0.999(0.998-1.00)$ & $0.018^{*}$ \\
\hline Geographic region & & & N/A \\
\hline Europe, Northern America & 20 & 1.00 & \\
\hline Latin America and the Caribbean & 66 & $7.90(1.66-37.6)$ & $0.009^{*}$ \\
\hline Northern Africa, Western Asia & 67 & $11.9(1.82-78.0)$ & $0.010^{*}$ \\
\hline Southern Africa & 62 & $9.72(1.85-51.2)$ & $0.007^{*}$ \\
\hline Western Africa, Middle Africa & 79 & $15.5(3.50-68.7)$ & $<0.001^{*}$ \\
\hline Eastern Africa & 84 & $18.5(4.08-83.8)$ & $<0.001^{*}$ \\
\hline Eastern Asia, Central Asia & 56 & $6.41(0.930-44.2)$ & 0.059 \\
\hline South-Eastern Asia & 87 & $21.2(4.48-100)$ & $<0.001^{*}$ \\
\hline Southern Asia & 91 & $32.4(6.24-168)$ & $<0.001^{*}$ \\
\hline Duration of travel, days ${ }^{b}$ & N/A & $1.025(1.00-1.05)$ & $0.048^{*}$ \\
\hline Accommodation & & & N/A \\
\hline hotel & 68 & 1.00 & \\
\hline home of a local & 87 & $2.36(0.922-6.06)$ & 0.073 \\
\hline guest house & 87 & $1.78(0.940-3.36)$ & 0.077 \\
\hline \multicolumn{4}{|l|}{ Eating uncooked meat/fish } \\
\hline did not eat uncooked meat/fish & 81 & 1.00 & \\
\hline ate uncooked meat/fish & 66 & $0.303(0.147-0.625)$ & $0.001^{*}$ \\
\hline
\end{tabular}

Abbreviations: $\mathrm{AOR}$ adjusted odds ratios, $\mathrm{Cl}$ confidence interval, N/A not applicable

a Backward selection eliminated the following factors: location, other close contact with local, having insect stings, and use of utensils

${ }^{\mathrm{b}}$ Analyzed as continuous variables. Age also on quadratic term, which seems to be better than age only in the model by AIC

${ }^{\mathrm{c}} 10$ datasets used in imputation

At follow-up, the four leading symptoms were fever, skin problems, respiratory illness, and TD, a finding according with Hill's results [4]. In our study, fever was the most frequent single symptom - often associated with TD. Diarrhea remained the most common cause for seeking health care.

\section{Profiles}

The data revealed regional differences which enabled profiling our subjects: travelers to Africa tended to be older and more cautious than those visiting Asia, whereas visitors to Asia were typically younger and favored longer trips. Likewise, the data show differences between travelers by age: the young traveled for longest and stayed in guest houses more frequently than the others, were the most likely to eat uncooked meat/fish and salads, not to use utensils, to follow a vegetarian diet, to neglect hand washing, and not to avoid freshwater contact. Not surprisingly, the longer the stay overseas, the lower the degree of travelers' compliance with hygiene instructions. Such traveler profiles can be used as a tool to target advice at various groups according to their special characteristics.

\section{Risk factors}

Destination, gender, age, and duration of travel were shown by multivariable analysis to be factors predisposing to illness. Southern Asia proved the riskiest resort, as also reported in earlier studies $[4,26]$. Female travelers proved to be at greater risk of acquiring symptoms (OR 1.7), a finding according with the results published by Hill [4]. In the present data, each day increased the risk of 


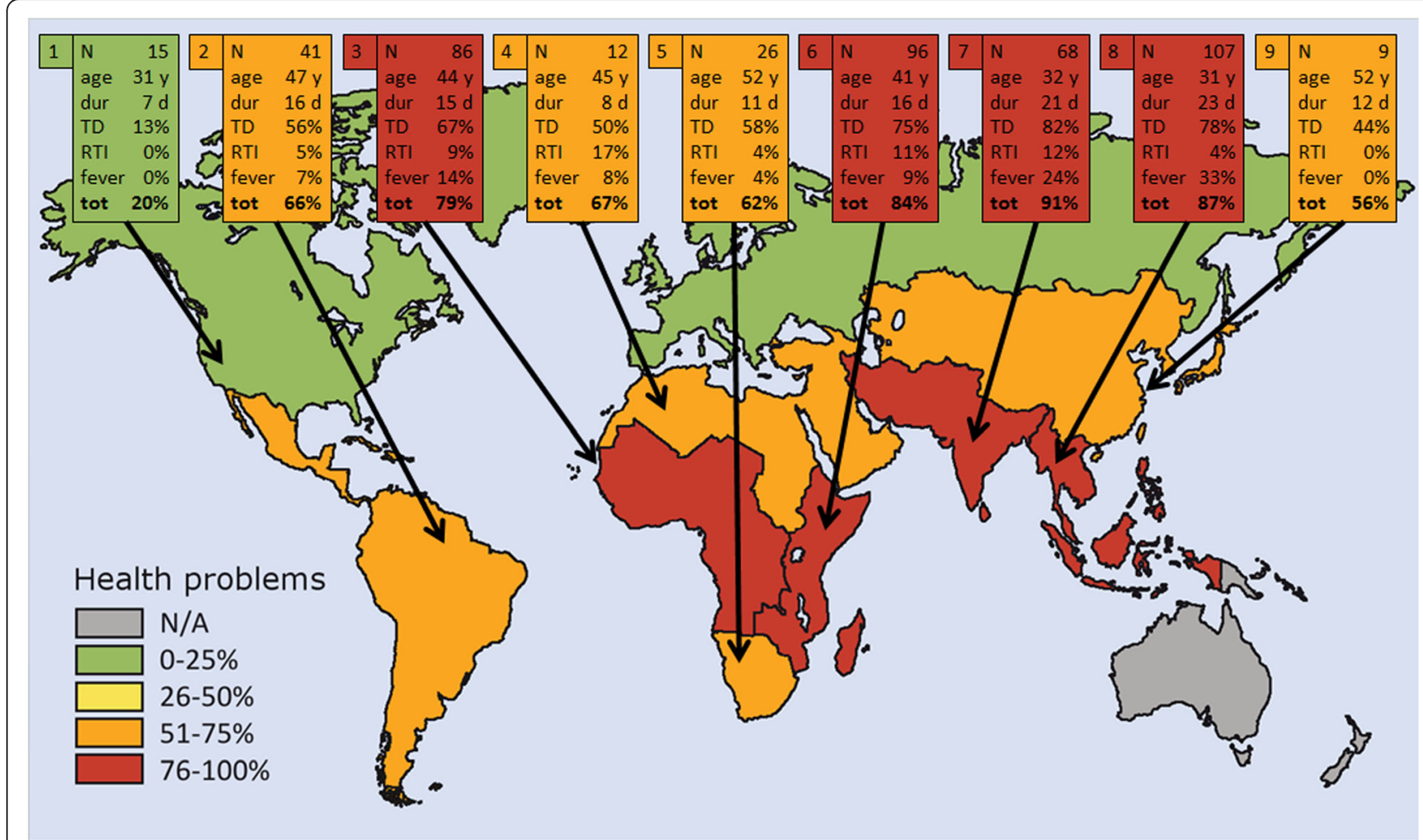

Fig. 2 Demographics and illnesses of the 460 travelers. Each box presents data from one of the nine destinations by reporting the number of visitors, their median age in years, median duration of travel in days, and percentages of traveler with diarrhea (TD), respiratory tract infection (RTI), fever, or any symptom while traveling / on arrival. Map graphics devised by Helena Schmidt, HumanArt. N/A = not applicable.1 Europe and Northern America. 2 Latin America and the Caribbean. 3 Western Africa and Middle Africa. 4 Northern Africa and Western Asia. 5 Southern Africa. 6 Eastern Africa. 7 Southern Asia. 8 South-Eastern Asia. 9 Eastern Asia and Central Asia

contracting illness by $2.5 \%$; the respective figure obtained by Hill was 3.1-3.7 \% [4]. Young age has often been reported to be associated with illness $[4,5,8,23,26$, 27]; in our cohort the risk was highest at 31.5 years. Eating raw meat or fish proved to be a protective factor. The reasons for this are not obvious and can only be speculated on.

\section{Limitations of the study}

The present investigation has some limitations which deserve to be discussed. Firstly, the results are not representative of all travelers, but of volunteers with pretravel appointments at a travel clinic. Thus visitors to Africa and other developing regions were overrepresented. Secondly, due to small sample size, statistical analyses of some subgroups were poorly powered. The third limitation concerns the follow-up: the reliability of the conclusions suffers from the fact that only $51 \%$ of our volunteers completed the questionnaire, and many delivered it later than requested. Importantly, however, even if it could be assumed that none of the remaining $49 \%$ had been ill, the number of newly onset symptoms during follow-up remains substantial. The data would have benefited from a comparison to non-traveling controls. Our data collection can also be regarded as a limitation, for questionnaire-based studies may distort the results in various ways. Due to the common use of questionnaires, this tends to be characteristic of research into travelers' health problems $[3,4,6,22-27,42,44-$ 48]; a few previous reports include data collected by telephone surveys [2, 4, 5, 8, 27]. In our study, travel diaries may have improved the accuracy of data to some degree. The exact number of clients declining to participate was not recorded. However, based on the number of clients seen by the recruiting doctor and number of those recruited, we estimate that at the maximum $10 \%$ of the potentially eligible clients declined.

\section{Our data in relation to previous estimates of travelers' health problems}

We recently reported incidences of illness and injury among more than 50000 Finnish travelers visiting various regions [21] by relating cases recorded by an assistance organization to numbers of travelers to each region. The results were considered to cover the most severe cases. The present data complete that picture by showing even the mildest symptoms contracted. This puts the two studies into perspective. Since $90 \%$ of our travelers did not contact health 
Table 7 Symptoms and contacts to health care providers. Data are provided for 459 travelers as numbers and percentages separately for the period during travel, on return, and one month after it

\begin{tabular}{|c|c|c|c|}
\hline & During journey, $\mathrm{N}$ & Ongoing or started on arrival, $\mathrm{N}$ & Newly onset during follow-up, $\mathrm{N}$ \\
\hline & $\overline{N / 459\left(\%{ }^{a}\right)}$ & $\overline{N / 453\left(\%^{a}\right)}$ & $\bar{N} / 233\left(\%^{\mathrm{a}}\right)$ \\
\hline Any symptom & $351(76)$ & $113(25)$ & $74(32)$ \\
\hline travelers' diarrhea & $312(68)$ & $78(17)$ & $5(2)$ \\
\hline skin problem ${ }^{b}$ & $78(17)$ & $2(0)$ & $9(4)$ \\
\hline fever $>37,5^{\circ} \mathrm{C}$ & $74(16)$ & $6(1)$ & $20(9)$ \\
\hline vomiting & $55(12)$ & $2(0)$ & $1(0)$ \\
\hline respiratory tract infection & $26(6)$ & $17(4)$ & $9(4)$ \\
\hline urinary tract infection & $17(4)$ & $1(0)$ & $4(2)$ \\
\hline ear infection & $5(1)$ & $4(1)$ & $1(0)$ \\
\hline other gastrointestinal symptoms & $19(4)$ & $2(0)$ & $4(2)$ \\
\hline other symptoms & $16(3)$ & $6(1)$ & $40(17)$ \\
\hline Contacts to health care & $47(10)$ & $1(0)$ & $50(21)$ \\
\hline Outpatient contact with health care ${ }^{c}$ & $44(10)$ & $1(0)$ & $46(20)$ \\
\hline travelers' diarrhea & $20(4)$ & $0(0)$ & $16(7)$ \\
\hline skin problem ${ }^{d}$ & $9(2)$ & $0(0)$ & $5(2)$ \\
\hline respiratory tract infection & $8(2)$ & $0(0)$ & $5(2)$ \\
\hline ear infection & $4(1)$ & $0(0)$ & $1(0)$ \\
\hline malaria / suspected malaria & $3(1)$ & $0(0)$ & $0(0)$ \\
\hline musculosceletal problem & $2(0)$ & $0(0)$ & $6(3)$ \\
\hline urinary tract infection & $2(0)$ & $0(0)$ & $2(1)$ \\
\hline other reason & $3(1)$ & $1(0)$ & $11(5)$ \\
\hline Inpatient (hospitalization) & $3(1)$ & $0(0)$ & $4(2)$ \\
\hline travelers' diarrhea & $2(0)$ & $0(0)$ & $1(0)$ \\
\hline fever $>37,5^{\circ} \mathrm{C}$ & $0(0)$ & $0(0)$ & $1(0)$ \\
\hline respiratory tract infection & $0(0)$ & $0(0)$ & $1(0)$ \\
\hline suspected malaria & $0(0)$ & $0(0)$ & $1(0)$ \\
\hline other reason & $1(0)$ & $0(0)$ & $0(0)$ \\
\hline
\end{tabular}

a Proportion of positive among those from whom information was available

b Dry skin / atopy / acne / insect stings excluded, sunburn / sun rash / infected skin due to insect stings included

c Five travelers reported two or more causes of visit

${ }^{d}$ Including travelers seeing a doctor because of any skin problem (e.g. insect stings, allergic reactions)

care, the proportion covered by our previous report may not amount to more than $10 \%$ of the illness altogether slightly less than estimated in that study [21]. The strengths of the present research include the prospective study design and the fact that we combined data of three time points (pre-travel, travel/return, and follow-up) for a single cohort. Interestingly, the rate of health problems per region presented in our study accords with risk estimates presented by International SOS (Additional file 2: Figure S2).

\section{Aspects related to malaria prophylaxis and treatment}

Advice on malaria prophylaxis is a cornerstone of pretravel appointments. In our data, 289 of the 296 travelers (98\%) taking malaria prophylaxis reported compliance. Interestingly, however, four of them, all diagnosed in
Africa, were also treated for malaria while abroad. One case was microbiologically verified, two reported a negative malaria test, and one had taken medication without laboratory diagnostics. Our earlier data collected by the assistance organization shows malaria to be rare in travelers $(8 / 50000$ cases; Siikamäki, personal communication). Indeed, the diagnoses of our cases may not be correct, as presumptive treatment is often given in Africa [49], and malaria diagnostics may not always be accurate [50].

\section{Conclusions}

This study with most of its subjects visiting (sub)tropical regions shows that, despite efficient preventive measures like vaccinations, malaria prophylaxis, and travel advice, the majority fall ill during or after 
travel. TD is the most common disease while abroad, followed by skin problems and fever. After travel, the most frequent complaints are fever, respiratory tract infections, and skin problems. Symptoms generally remain mild, not requiring medical care. The proportion of newly onset illness among returning travelers is considerable: one-third get health problems after their journeys. Advice regarding this should be given already at pre-travel appointments.

\section{Additional files}

Additional file 1: Figure S1. HealthMap 2010 by International SOS. Medical risk ratings are based on the standard of local medical and dental care, access to prescription drugs, the possible prevalence of serious infectious diseases, and known cultural, linguistic and administrative barriers. Map printed with the written permission of International SOS. This map has been developed for illustrative purposes only. It is a global illustration of medical risk for travellers. For detailed information, please refer to the country guides at internationalsos.com (c) International SOS, 2010. All rights reserved. Unauthorized copy or distribution prohibited. (PDF $3143 \mathrm{~kb}$ )

Additional file 2: Figure S2. HealthMap 2010 by International SOS presented together with demographics and illnesses of our 460 travelers. Each box presents data from one of the nine destinations by reporting the number of visitors, their median age in years, median duration of travel in days, and percentages of traveler with diarrhea (TD), respiratory tract infection (RTI), fever, or any symptom while traveling / on arrival. Map printed and modified with the written permission of International SOS. This map has been developed for illustrative purposes only. It is a global illustration of medical risk for travellers. For detailed information, please refer to the country guides at internationalsos.com ( I) International SOS, 2010. All rights reserved. Unauthorized copy or distribution prohibited. 1 Europe and Northern America. 2 Latin America and the Caribbean. 3 Western Africa and Middle Africa. 4 Northern Africa and Western Asia. 5 Southern Africa. 6 Eastern Africa. 7 Southern Asia. 8 South-Eastern Asia. 9 Eastern Asia and Central Asia. (TIF 604 kb)

\section{Abbreviations}

TD, Travelers' diarrhea

\section{Acknowledgements}

We express our gratitude to the late Dr Jukka Riutta for recruiting the patients. We thank the nurses at the Travel Clinic of Aava Medical Centre for help in recruiting the volunteers, and the personnel of Helsinki University Hospital Laboratory for assistance with the collection of questionnaires. Jukka Ollgren (National Institute for Health and Welfare, Helsinki, Finland) is acknowledged for advice in statistical analyses.

\section{Funding}

The work was supported by the Finnish Governmental Subsidy for Health Science Research and by the Scandinavian Society for Antimicrobial Chemotherapy Foundation. The funding sources had no involvement in study design, data collection, analysis, data interpretation, writing of the report, and the decision to submit the article for publication.

\section{Availability of data and materials}

Data not available.

\section{Authors' contributions}

Study concept and design, KV, AK; acquisition of data, KV, SHP, TL, AK; analysis and interpretation of data, KV, SHP, TL, AK; statistical analysis, KV; drafting of the manuscript, KV, AK; critical comments on the manuscript, SHP,
TL, HS; final approval of the version published, KV, SHP, TL, HS, AK. All authors have read and approved the final version of the manuscript.

\section{Authors' information}

Not applicable.

\section{Competing interests}

The authors declare that they have no competing interests.

\section{Consent for publication}

Not applicable.

Ethics approval and consent to participate

The study protocol was approved by the Ethics Committee of Helsinki University Hospital. Written informed consent was obtained from all subjects.

\section{Author details}

${ }^{1}$ Department of Bacteriology and Immunology, University of Helsinki, Haartmaninkatu 3, (P.O. Box 21), 00014 Helsinki, Finland. Inflammation Center, Clinic of Infectious Diseases, Helsinki University Hospital and University of Helsinki, Aurora Hospital, Nordenskiöldinkatu 20, (P.O. Box 348), Helsinki, Finland. ${ }^{3}$ Institute of Clinical Medicine, University of Helsinki, Helsinki, Finland. ${ }^{4}$ Aava Travel Clinic, Medical Centre Aava, Annankatu 32, 00100 Helsinki, Finland. ${ }^{5}$ Unit of Infectious Diseases, Solna, Karolinska Institutet, SE-171 76 Stockholm, Sweden.

Received: 6 January 2016 Accepted: 20 June 2016

Published online: 13 July 2016

\section{References}

1. United Nations World Tourism Organization (UNWTO). Available at: http://cf. cdn.unwto.org/sites/all/files/pdf/unwto_barom16_01_january_excerpt.pdf. Accessed 7 Jul 2016.

2. Duval B, De Serre G, Shadmani R, Boulianne N, Pohani G, Naus M, et al. A population-based comparison between travelers who consulted travel clinics and those who did not. J Travel Med. 2003:10(1):4-10.

3. Wilder-Smith A, Khairullah NS, Song JH, Chen CY, Torresi J. Travel health knowledge, attitudes and practices among Australasian travelers. J Travel Med. 2004;11(1):9-15.

4. Hill DR. Health problems in a large cohort of Americans traveling to developing countries. J Travel Med. 2000;7(5):259-66.

5. Winer L, Alkan M. Incidence and precipitating factors of morbidity among Israeli travelers abroad. J Travel Med. 2002;9(5):227-32.

6. Kemmerer TP, Cetron M, Harper L, Kozarsky PE. Health problems of corporate travelers: risk factors and management. J Travel Med. 1998;5(4): 184-7.

7. Freedman DO, Weld LH, Kozarsky PE, Fisk T, Robins R, von Sonnenburg F, et al. Spectrum of disease and relation to place of exposure among ill returned travelers. N Engl J Med. 2006;354(2):119-30.

8. Alon D, Shitrit P, Chowers M. Risk behaviors and spectrum of diseases among elderly travelers: a comparison of younger and older adults. J Travel Med. 2010;17(4):250-5

9. Siikamaki HM, Kivela PS, Sipila PN, Kettunen A, Kainulainen MK, Ollgren JP, et al. Fever in travelers returning from malaria-endemic areas: don't look for malaria only. J Travel Med. 2011;18(4):239-44.

10. Flores-Figueroa J, Okhuysen PC, von Sonnenburg F, DuPont HL, Libman MD, Keystone JS, et al. Patterns of illness in travelers visiting Mexico and Central America: the GeoSentinel experience. Clin Infect Dis. 2011;53(6):523-31.

11. Savini H, Gautret P, Gaudart J, Field V, Castelli F, Lopez-Velez R, et al. Travelassociated diseases, Indian Ocean Islands, 1997-2010. Emerg Infect Dis. 2013. 19(8):1297-301

12. Harvey K, Esposito DH, Han P, Kozarsky P, Freedman DO, Plier DA, et al. Surveillance for travel-related disease-GeoSentinel Surveillance System, United States, 1997-2011. MMWR Surveill Summ. 2013;62:1-23.

13. Leder K, Torresi J, Brownstein JS, Wilson ME, Keystone JS, Barnett E, et al. Travel-associated illness trends and clusters, 2000-2010. Emerg Infect Dis. 2013;19(7):1049-73.

14. Leder K, Torresi J, Libman MD, Cramer JP, Castelli F, Schlagenhauf P, et al. GeoSentinel surveillance of illness in returned travelers, 2007-2011. Ann Intern Med. 2013;158(6):456-68. 
15. Boggild AK, Geduld J, Libman M, Ward BJ, McCarthy AE, Doyle PW, et al. Travel-acquired infections and illnesses in Canadians: surveillance report from CanTravNet surveillance data, 2009-2011. Open Med. 2014;8(1):e20-32.

16. Hagmann SH, Han PV, Stauffer WM, Miller AO, Connor BA, Hale DC, et al. Travel-associated disease among US residents visiting US GeoSentinel clinics after return from international travel. Fam Pract. 2014;31(6):678-87.

17. Wilson ME, Chen LH, Han PV, Keystone JS, Cramer JP, Segurado A, et al. Illness in travelers returned from Brazil: the GeoSentinel experience and implications for the 2014 FIFA World Cup and the 2016 Summer Olympics. Clin Infect Dis. 2014:58(10):1347-56.

18. Mendelson M, Han PV, Vincent P, von Sonnenburg F, Cramer JP, Loutan L, et al. Regional variation in travel-related illness acquired in Africa, March 1997-May 2011. Emerg Infect Dis. 2014;20(4):532-41.

19. Al-Abri SS, Abdel-Hady DM, Al Mahrooqi SS, Al-Kindi HS, Al-Jardani AK, Al-Abaidani IS. Epidemiology of travel-associated infections in Oman 1999-2013: A retrospective analysis. Travel Med Infect Dis. 2015;13(5):388-93.

20. Boggild AK, Esposito DH, Kozarsky PE, Ansdell V, Beeching NJ, Campion D, et al. Differential diagnosis of illness in travelers arriving from Sierra Leone, Liberia, or Guinea: a cross-sectional study from the GeoSentinel Surveillance Network. Ann Intern Med. 2015;162(11):757-64.

21. Siikamaki H, Kivela P, Fotopoulos M, Ollgren J, Kantele A. Illness and injury of travellers abroad: Finnish nationwide data from 2010 to 2012, with incidences in various regions of the world. Euro Surveill. 2015;20(19):15-26.

22. Getz L, Larssen KE, Dahl B, Westin S. Health problems in Norwegians travelling to distant countries. Scand J Prim Health Care. 1990;8(2):95-100.

23. Ahlm C, Lundberg S, Fesse K, Wistrom J. Health problems and selfmedication among Swedish travellers. Scand J Infect Dis. 1994;26(6):711-7.

24. Bruni M, Steffen R. Impact of Travel-Related Health Impairments. J Travel Med. 1997;4(2):61-4.

25. Evans MR, Shickle D, Morgan MZ. Travel illness in British package holiday tourists: prospective cohort study. J Infect. 2001;43(2):140-7.

26. Rack J, Wichmann O, Kamara B, Gunther M, Cramer J, Schonfeld C, et al. Risk and spectrum of diseases in travelers to popular tourist destinations. J Travel Med. 2005;12(5):248-53.

27. Fleck S, Jager H, Zeeb H. Travel and health status: a survey follow-up study. Eur J Public Health. 2006:16(1):96-100.

28. Kantele A, Laaveri T, Mero S, Vilkman K, Pakkanen SH, Ollgren J, et al. Antimicrobials increase travelers' risk of colonization by extended-spectrum betalactamase-producing Enterobacteriaceae. Clin Infect Dis. 2015;60(6):837-46.

29. Antikainen J, Kantele A, Pakkanen SH, Laaveri T, Riutta J, Vaara M, et al. A quantitative polymerase chain reaction assay for rapid detection of 9 pathogens directly from stools of travelers with diarrhea. Clin Gastroenterol Hepatol. 2013;11(10):1300-7. e3.

30. Laaveri T, Antikainen J, Pakkanen SH, Kirveskari J, Kantele A: Prospective study of pathogens in asymptomatic travellers and those with diarrhoea: aetiological agents revisited. Clin Microbiol Infect 2016, in press.

31. http://www.who.int/topics/diarrhoea/en/. Accessed 7 Jul 2016.

32. http://unstats.un.org/unsd/methods/m49/m49regin.htm. Accessed 7 Jul 2016.

33. http://www.un.org/en/development/desa/policy/wesp/wesp_current/ 2012country_class.pdf. Accessed 7 Jul 2016.

34. Mattila L, Siitonen A, Kyronseppa H, Simula II, Peltola H. Risk Behavior for Travelers' Diarrhea Among Finnish Travelers. J Travel Med. 1995;2(2):77-84.

35. Steffen R, Tornieporth N, Clemens SA, Chatterjee S, Cavalcanti AM, Collard F, et al. Epidemiology of travelers' diarrhea: details of a global survey. J Travel Med. 2004;11(4):231-7.

36. Cabada MM, Maldonado F, Quispe W, Mozo K, Serrano E, Gonzalez E, et al. Risk factors associated with diarrhea among international visitors to Cuzco, Peru. Am J Trop Med Hyg. 2006;75(5):968-72.

37. Laverone E, Boccalini S, Bechini A, Belli S, Santini MG, Baretti S, et al. Travelers' compliance to prophylactic measures and behavior during stay abroad: results of a retrospective study of subjects returning to a travel medicine center in Italy. J Travel Med. 2006;13(6):338-44.

38. Piyaphanee W, Kusolsuk T, Kittitrakul C, Suttithum W, Ponam T, Wilairatana $P$. Incidence and impact of travelers' diarrhea among foreign backpackers in Southeast Asia: a result from Khao San road, Bangkok. J Travel Med. 2011;18(2):109-14.

39. Kasper MR, Lescano AG, Lucas C, Gilles D, Biese BJ, Stolovitz G, et al. Diarrhea outbreak during U.S. military training in El Salvador. PLoS One. 2012;7(7):e40404.
40. Launders NJ, Nichols GL, Cartwright R, Lawrence J, Jones J, Hadjichristodoulou C. Self-reported stomach upset in travellers on cruisebased and land-based package holidays. PLoS One. 2014;9(1):e83425.

41. Kittitrakul C, Lawpoolsri S, Kusolsuk T, Olanwijitwong J, Tangkanakul W, Piyaphanee W. Traveler's Diarrhea in Foreign Travelers in Southeast Asia: A Cross-Sectional Survey Study in Bangkok, Thailand. Am J Trop Med Hyg. 2015;93(3):485-90.

42. Reid D, Dewar RD, Fallon RJ, Cossar JH, Grist NR. Infection and travel: the experience of package tourists and other travellers. J Infect. 1980;2(4):365-70.

43. Schlagenhauf $P$, Chen LH, Wilson ME, Freedman DO, Tcheng D, Schwartz E, et al. Sex and gender differences in travel-associated disease. Clin Infect Dis. 2010;50(6):826-32

44. Mackaness CA, Osborne A, Verma D, Templer S, Weiss MJ, Knouse MC. A quality improvement initiative using a novel travel survey to promote patient-centered counseling. J Travel Med. 2013;20(4):237-42.

45. Reed JM, McIntosh IB, Powers K. Travel IIlness and the Family Practitioner: A Retrospective Assessment of Travel-Induced IIIness in General Practice and the Effect of a Travel IIIness Clinic. J Travel Med. 1994;1(4):192-8.

46. Lee VJ, Wilder-Smith A. Travel characteristics and health practices among travellers at the travellers' health and vaccination clinic in Singapore. Ann Acad Med Singapore. 2006;35(10):667-73.

47. Goesch JN, Simons de Fanti A, Bechet S, Consigny PH. Comparison of knowledge on travel related health risks and their prevention among humanitarian aid workers and other travellers consulting at the Institut Pasteur travel clinic in Paris, France. Travel Med Infect Dis. 2010;8(6):364-72.

48. Buhler S, Ruegg $\mathrm{R}$, Steffen $\mathrm{R}$, Hatz C, Jaeger VK. A profile of travelers-an analysis from a large swiss travel clinic. J Travel Med. 2014;21(5):324-31.

49. Vialle-Valentin CE, LeCates RF, Zhang F, Ross-Degnan D. Treatment of Febrile illness with artemisinin combination therapy: prevalence and predictors in five African household surveys. J Pharm Policy Pract. 2015;8(1):1. 014-0024-0. eCollection 2015.

50. Kahama-Maro J, D'Acremont V, Mtasiwa D, Genton B, Lengeler C. Low quality of routine microscopy for malaria at different levels of the health system in Dar es Salaam. Malar J. 2011;10:332. 2875-10-332.

\section{Submit your next manuscript to BioMed Central and we will help you at every step:}

- We accept pre-submission inquiries

- Our selector tool helps you to find the most relevant journal

- We provide round the clock customer support

- Convenient online submission

- Thorough peer review

- Inclusion in PubMed and all major indexing services

- Maximum visibility for your research

Submit your manuscript at www.biomedcentral.com/submit 